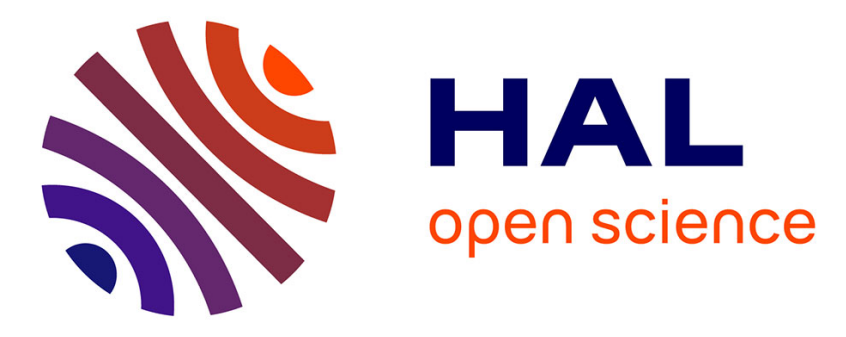

\title{
Contribution of actuated head and trunk to passive walkers stabilization
}

\author{
Mehdi Benallegue, Jean-Paul Laumond, Alain Berthoz
}

\section{To cite this version:}

Mehdi Benallegue, Jean-Paul Laumond, Alain Berthoz. Contribution of actuated head and trunk to passive walkers stabilization. IEEE, International Conference on Robotics and Automation, May 2013, Karlsruhe, France. pp.5638 - 5643, 10.1109/ICRA.2013.6631387 . hal-01079775

\section{HAL Id: hal-01079775 \\ https://hal.science/hal-01079775}

Submitted on 3 Nov 2014

HAL is a multi-disciplinary open access archive for the deposit and dissemination of scientific research documents, whether they are published or not. The documents may come from teaching and research institutions in France or abroad, or from public or private research centers.
L'archive ouverte pluridisciplinaire HAL, est destinée au dépôt et à la diffusion de documents scientifiques de niveau recherche, publiés ou non, émanant des établissements d'enseignement et de recherche français ou étrangers, des laboratoires publics ou privés. 


\title{
Contribution of Actuated Head and Trunk to Passive Walkers Stabilization
}

\author{
Mehdi Benallegue*, Jean-Paul Laumond ${ }^{\dagger}$, Alain Berthoz* \\ *Laboratoire de Physiologie de la Perception et de l'Action, UMR 7152 CNRS / Collège de France. \\ 11 place Marcelin Berthelot 75005 Paris, France. Email: \{mehdi.benallegue, alain.berthoz\}@ college-de-france.fr \\ ${ }^{\dagger}$ LAAS-CNRS, University of Toulouse, 7 avenue du Colonel Roche 31077 Toulouse, France. Email: jpl@laas.fr
}

\begin{abstract}
Passivity-based walkers represent a model for human walking and a solution for low-energy locomotion for humanoid robots. The presence of an upper-body and even a head in this kind of systems is necessary as a better model for humans and to improve their usability. The benefits of these additions have never been studied, and no work experimented the addition of a head limb to a passivity-based walker. So, we aim, in this paper, to study the effects of the addition of these modifications on walkers with fully passive lower limbs. By comparing three systems (a passive compass, an upper-body stabilizing walker and a head stabilizing walker) simulations show that: (ii) upper-body stabilization improves the stability of the walking limit cycle; (ii) in return, the stabilization of the upper body requires a noticeable amount of the kinetic energy of the walker, and a significant energy supply (steeper slopes for the passive case) is necessary to guarantee the stability of the gait, especially for the case of head stabilization; and (iii) in a dynamical context, such as steep slopes, the upper-body and head stabilization have close performances for absorbing perturbations and smoothing the impacts, but with a slight advantage for the latter.
\end{abstract}

\section{INTRODUCTION}

Legged, and especially biped, locomotion is acknowledged to be the most adapted moving technique when sharing the same space as humans. However, there lies an intrinsic falling hazard in the dynamics a walker. A robot's state is said to be viable if the robot is able to avoid the fall [1]. Walking is then a very dynamic task which requires energy. However, an important parameter for biped robot is the energy-efficiency of walking, and the robots that show the best efficiency are passive walking robots. Indeed, on shallow slopes, very simple mechanical designs with appropriate mass distributions show balanced and durable gaits with no energy source other than the gravity. This efficiency property was then adapted to generate low power walking systems on level ground by the use of simple actuators to compensate gravity [2]. Moreover, passive walkers are commonly assumed to generate more human-like motions, even with the presence of geometrical or mechanical discrepancies between the robot and humans [3]. This similarity has attracted the interest of biomechanics to study the passivity of the human gait [4].

In order to improve the accuracy of this model for humans, the presence of a vertical upper-body is crucial, because the dynamics and mass contributions of the human's upper-body are important. Moreover, any biped robot that is intended to have actual usability other than a simple locomotion requires to have an upper body, for example to enable manipulation and interaction. Indeed, some researches have studied the addition of an upper-body to a passive walker, but none of them, to our knowledge, studied the effect of a vertical stabilization of this upper-body on the stability of the limit-cycle, nor the benefits on the set of viable states.

One other important feature of human walking, that does not enter into account within the aforementioned researches, is the role of the head and gaze in human locomotion. Indeed, it has been shown that during walking motions, the head is stabilized in rotation [5]. This stabilization is a general phenomenon observed for several human tasks and is believed to enable a more stable reference frame for egocentric visual motion perception and a more consistent visual-vestibular sensory inputs. In the same spirit, it has been shown that the head stabilization provides a major improvement of the estimation of the vertical direction for robots, particularly when relying on a vestibular-like inertial measurement system [6]. An accurate estimation of the vertical is considered as a crucial parameter to ensure balance for humans and humanoid robots. However, no research, to our best knowledge, studied the possibility to add a head link to a passive walker.

We propose to study, in a pure mechanical point-of-view, the benefits of upper-body and head stabilization on the simplest human-like walking systems: a purely passive lowerbody. The paper aims particularly to study the case of the appearance of unexpected perturbations during cyclic walking. We propose then to compare the simulated dynamics of three models: (i) the classical compass walker (ii) a walker with a stabilized simple upper-body, and (iii) a novel passive walker model with an upper body and a head, both stabilized.

In the following section, we describe these three models, their control and our implementation. In the section III, we describe the results we obtained when evaluating the viability kernels of these walkers for different slope inclinations. And in the section IV, we show an in-depth analysis of the cyclic walking on shallow slope.

\section{WALKING SYSTEMS}

\section{A. The mechanical model}

We study in this paper two main 2D models for passive walkers: one with only two links (compass) and the other one equipped with upper-body segments, as depicted in Fig 1 . The walker has two massless legs of $l_{p}$ length, with, on each leg, 
a point mass of $m_{l}$ at $l_{l}$ distance to the joint between them. The trunk is a masseless stick of $l_{t}$ length, linked to legs, and having a point mass of $m_{t}$ on its middle. The head is a point mass of $m_{h}$ on the top of a stick of $l_{h}$ length, linked to the torso. To keep the simplicity of the model, we didn't study the case of the presence of knees, ankles or arms even if they have noticeable effects on the dynamics of the walker [7]-[9].

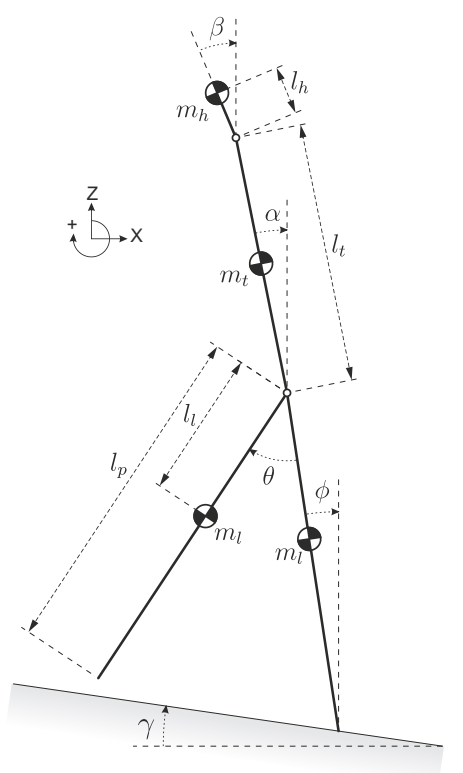

Figure 1. Our walker model. The mass on the trunk is at the middle of the trunk joint. The case of two segment compass corresponds to $l_{t}=l_{h}=0$.

There are two major ways to add an upper body to a passive walker. First, the bisector constrained walker, introduced by Wisse et al [10], is a compass with an upper limb that is constrained to be in the midway angle of the two legs. However, beside the fact that it is not an accurate model of human walking, its bisecting constraint introduces an instability, especially in the presence of a big upper body mass [11], which is the case of humans. Therefore, none of our simulations succeeded to show any viable state for this model. The second method is to stabilize actively the upper body against the vertical. This method better models the human gait. The stabilization is achieved commonly by applying torque on the stance leg [12] which has the advantage to not disturb the passivity of the swing motion. The torque applied on the stance leg is denoted $\tau_{t}$. The head stabilization is ensured by another pure torque generator in the neck link, between the head and the trunk. This torque is denoted $\tau_{h}$.

The walker has always a stance leg on the ground. That makes the compass position fully describable with four angles: the inter-legs angle $(\theta)$ and the stance leg, torso and head inclinations with regard to vertical ( $\phi, \alpha$ and $\beta$ respectively).

We study also the case where there is no upper body, which is equivalent in our case to consider $l_{t}=l_{h}=0$. In that case the angles $\alpha$ and $\beta$ have no influence on the dynamics of the compass and can be ignored.

\section{B. The dynamics state}

The dynamics state $x$ of the walker can be defined by the limbs angles and their angular velocities $x=$ $\left[\begin{array}{llllllll}\theta & \phi & \alpha & \beta & \dot{\theta} & \dot{\phi} & \dot{\alpha} & \dot{\beta}\end{array}\right]^{t}$. The evolution of the state over time is continuous except in impacts. The impacts on the ground are considered perfectly inelastic and stiff. That introduces discontinuities in the angular velocities, and the stance leg switch introduces a change in the sign of $\theta$. Therefore, we prefer a discreet time expression of the evolution of the dynamics state. We consider the future discretized state $x_{k+1}$ as completely determined by the previous state $x_{k}$ and the torques vector $\tau_{k}=\left[\begin{array}{ll}\tau_{t, k} & \tau_{h, k}\end{array}\right]^{t}$ :

$$
x_{k+1}=f\left(x_{k}, \tau_{k}\right)
$$

The function $f$ integrates the dynamics equations over sampling time. These equations won't be detailed here and can be adapted from former works [13]. We used a software dynamic simulator to compute the dynamics and detect contacts during walking motions. We consider that contacts always happen on a sampling instant, and that the dynamics state during this instant is the pre-contact one. The contacts are considered without slipping or deformation. The falling state is detected in three cases: when (i) there is no contact on the ground, (ii) there is a contact with a limb other than a foot, or (iii) a double support lasts for more than one instant, which corresponds to a stop in the walking motion.

\section{The controllers}

The control has to be different from a walker model to another. For example for the simple two-links walker, the torques have no effect on the dynamics and are ignored. The walkers equipped with an upper body have to be actively controlled to maintain the trunk and the head above the ground.

1) Trunk stabilization controller: To control the robot with one-link upper-body, we add a rigid constraint on the neck joints to guarantee that $\alpha=\beta$. The proposed controller is a proportional derivative (PD) servoing the upper-body to be vertical, i.e. $\alpha=0$. The torque expression is then:

$$
\tau_{1, t}=-K_{1, p} \alpha-K_{1, d} \dot{\alpha}
$$

With $K_{1, p}$ and $K_{1, d}$ are the proportional and derivative gains of the controller. The controller behaves like an angular spring-damper on the trunk-to-stance joint. However, we cannot assume any actually realizable equivalent setting with a passive spring-damper because the vertical reference is defined in absolute frame and the stance leg changes at each step.

2) Head stabilization controller: The controller we suggest for the neck is also a PD bringing back $\beta$ to zero:

$$
\tau_{2, h}=-K_{2, p} \beta-K_{2, d} \dot{\beta}
$$

This walker requires also to control all the upper-body and maintain it upright. The controller we propose for trunk stabilization will try maintain the center of mass of the upper body $c_{u}$ above the hips. To do so, we use another PD to 
control the inclination $\mu$ of the line joining $c_{u}$ to the hips (see Fig. 2-C):

$$
\tau_{2, t}=-K_{3, p} \mu-K_{3, d} \dot{\mu}
$$
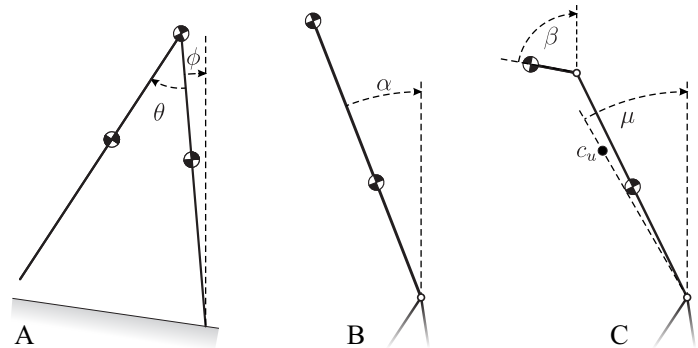

Figure 2. The three walker models. A- The two-links compass has no upper body and no actuator. B- The walker with a simple-trunk stabilization has only one link on the upper body. C- The upper body of the walker with a stabilized head has two links. The controller brings back $\mu$ to zero, i.e. the center of mass of the upper body $c_{u}$ vertically on the hips.

\section{The simulated walkers parameter values}

For the robots equipped with an upper-body, we have chosen to use a human-like mass distribution and body proportions [14], but by simplifying and rounding them for simulation reproducibility. Even if the two-links compass doesn't have an upper body, we kept the same masses distribution to guarantee a coherent comparison. The masses are: $m_{l}=1.5 \mathrm{~kg}, m_{t}=4.5 \mathrm{~kg}, m_{h}=0.5 \mathrm{~kg}$ and body lengths are $l_{p}=1 \mathrm{~m}, l_{l}=0.6 \mathrm{~m}$, and for upper-body equipped walkers: $l_{t}=0.75 \mathrm{~m}$ and $l_{h}=0.125 \mathrm{~m}$.

The controllers parameters were also fixed. For the trunk stabilization we set $K_{1, p}=30 \mathrm{~N} \cdot \mathrm{m} / \mathrm{rad}$ and $K_{1, d}=$ $15 \mathrm{~N} \cdot \mathrm{m} \cdot \mathrm{s} / \mathrm{rad}$. We limited the absolute value of the torque $\left|\tau_{t}\right|<50 \mathrm{~N} \cdot \mathrm{m}$. This value is not bio-inspired but only prevents the system from producing excessive torques. For the head stabilization, we choose to have the same upper body stabilization parameter, the controller parameters are then also $K_{3, p}=30 \mathrm{~N} \cdot \mathrm{m} / \mathrm{rad}, K_{3, d}=15 \mathrm{~N} \cdot \mathrm{m} \cdot \mathrm{s} / \mathrm{rad}$, with $\left|\tau_{t}\right|<$ $50 \mathrm{~N} \cdot \mathrm{m}$. For head stabilization, we took $K_{2, p}=5 \mathrm{~N} \cdot \mathrm{m} / \mathrm{rad}$ and $K_{2, d}=0.5 \mathrm{~N} \cdot \mathrm{m} \cdot \mathrm{s} / \mathrm{rad}$. The neck torque $\tau_{h}$ had two limitations: $\left|\tau_{h}\right|<10 \mathrm{~N} \cdot \mathrm{m}$ during the double support instant in order to absorb the impact, and $\left|\tau_{h}\right|<2 \mathrm{~N} \cdot \mathrm{m}$ otherwise. These values permit to enable the best impact absorption in this joint while keeping the torque reasonable during the rest of the time.

With these models, we can finally start the simulations.

\section{VIABILITY KERNEL VS ENERGY CONSUMPTION}

We aim in this first study to compare the robustness of the three walkers to a variety of initial conditions on different slope inclinations. Then, we define a relaxed definition of a viable state which is: a state from which the walker is able to perform at least 6 steps. Although this new definition does not guarantee the balance of the walker for all future steps, it is a good indicator of the viability kernel and has a fast enough computation to permit to check large number of states.
To compare the performances of these walkers, they have to start from the same initial state. However, the state vector itself is different from a walker to another. Indeed, the twolinks compass has a state vector with only relevant variables, $\left[\begin{array}{llll}\theta & \phi & \dot{\theta} & \dot{\phi}\end{array}\right]^{t}$. For the stabilized-trunk walker, there are also $\alpha$ and $\dot{\alpha}$. And for the stabilized-head walker there are $\beta$ and $\dot{\beta}$ also. Therefore, for the last two ones, we provide constant initial values for $\alpha, \dot{\alpha}, \beta$ and $\dot{\beta}$. For each walker, the initial state is its mean value during double support, after it reaches its limit-cycle on $4^{\circ}$ slope (this limit-cycle will be detailed in subsection IV-B). Specifically, for the trunk stabilized walker the values are $\alpha_{0}=\beta_{0}=0.0454 \mathrm{rad} \dot{\alpha}_{0}=\dot{\beta}_{0}=0.251 \mathrm{rad} / \mathrm{s}$. For the head stabilized walker, the values are $\alpha_{0}=0.0643 \mathrm{rad}$, $\beta_{0}=0.0028 \mathrm{rad}, \dot{\alpha}_{0}=0.289 \mathrm{rad} / \mathrm{s}$, and $\dot{\beta}_{0}=0.176 \mathrm{rad} / \mathrm{s}$. After this initialization, they have the natural dynamics during all the simulation. The choice of these parameters is coherent with our idea to study the effects of unexpected perturbations during cyclic walking: the walkers are supposed to be stable in their cycle before the beginning of the simulation.

All the starting states, in our simulations, are in double support phase, during the pre-impact instant. In that instant, the knowledge of the slope angle $\gamma$ and the inter-leg angle $\theta$ is sufficient to give the stance leg inclination $\phi$. The initial state is then simplified to three scalars: $\theta, \dot{\theta}$ and $\dot{\phi}$.

The simulations are run for five different slope inclinations: $2^{\circ}, 4^{\circ}, 6^{\circ}, 8^{\circ}$ and $10^{\circ}$. For each inclination and each walker model, we ran simulations for 8 millions different states, which corresponds to 200 different values for each of the three dimensions. These values were uniformly distributed within ranges of values that cover all the viable states we could simulate. These ranges are $[0,1.309] \mathrm{rad}$ for $\theta_{0},[-11.4,130] \mathrm{rad} / \mathrm{s}$ for $\dot{\theta}_{0}$, and $[0,8] \mathrm{rad} / \mathrm{s}$ for $\dot{\phi}_{0}$.

For each initial state, the walker starts the simulation at double support state and the steps it makes are counted. If it reaches 6 steps, the initial state is said to be viable, if it falls before, the state is non viable. The sampling period is of $5 \mathrm{~ms}$ and the simulations have a complete duration of 90 hours.

The most important result we compare in this simulation is the volume of the viability kernel by counting the number of viable states among the 8 million combinations. The results are shown in Fig. 3.

One striking property in this figure is the difference of results between shallow and more steep slopes. For less than $6^{\circ}$ slopes, the simple two legs compass has a bigger viability kernel than upper-body equipped ones, and for steeper ones the upper body gives better results than a simple compass. The analysis we provide for this result is that the upper body does stabilize the walking motion, but this stabilization absorbs the total kinematic energy of the walker. As the only external source of energy is gravity, shallow slopes do not provide enough energy to compensate the loss due to stabilization. The two legs compass is much more energy-efficient, because the only loss of energy happens during impacts. Impacts depend on the angle of incidence of the swing leg on the ground, and in shallow slopes, the steps are much smaller and impacts preserve more kinematic energy. 


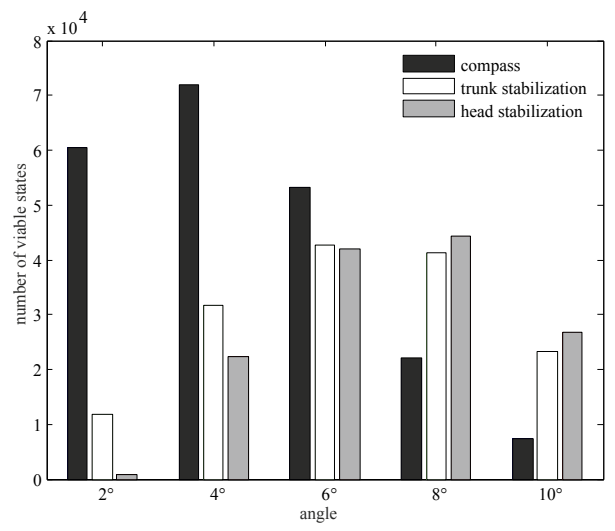

Figure 3. The volumes of viability kernels of the three models, for different slope inclinations (number of viable states among 8 million combinations).

On the contrary, in steeper slopes, the highly dynamic context provides kinematic energy on one hand, and an important instability on the other. Here can be demonstrated the stabilizing properties of upper-body control, especially on highly dynamic instability. We see then that the upper body stabilization absorbs an important part of this energy and helps to recover balance. Moreover, the head stabilization appears to be better again than the trunk only. The difference is tight, without surprise, because the mass contribution of the head is relatively less important. But we can say that there are states that are not viable for the trunk stabilization alone, and that can be recovered when the head is stabilized. The consequence of the control of the head is to distribute the stabilization of the upper body on more than a link. This result shows then that dividing the upper body on two (or more) hierarchically stabilized segments can improve the recovery for highly dynamical instability.

In this section, we have analyzed quantitatively the volume of viability kernel for the three walker models. We have seen that at $4^{\circ}$ slope, the two-links compass has a bigger viability kernel than the two other walker models. However, by analyzing deeper this specific result in the next section, we will see that the distribution of the viable states of a twolinks compass is not optimal regarding small perturbations to cyclic walking. We will see also that upper-body stabilization improves the stability of the walking limit cycle compared to a two-links compass.

\section{LiMIT CyCLE WALKING}

\section{A. Sensitivity to perturbations}

The context we are analyzing is particularly the recovery from unexpected perturbations. In such situation, the state of the walker is probably close to the limit cycle. From this perspective, we projected the viability kernels along the three dimensions, and studied their position compared to the limit cycle state. The mean limit cycle states are $\left[\begin{array}{lll}0.625 & 0.71 & 1.6\end{array}\right]^{t}$ for the compass, $\left[\begin{array}{lll}0.56 & -0.357 & 1.42\end{array}\right]^{t}$ for the trunk stabilization and $\left[\begin{array}{lll}0.54 & -0.23 & 1.41\end{array}\right]^{t}$ for the head stabilization.
The figures 4, 5 and 6 show the distribution of this 'error', where zero value means that the corresponding component of the state is the same as in the limit cycle. The densities have been normalized to have a total integral of $100 \%$. We see in Fig. 4 and 6 that the kernels are almost equally close to zero: the three models have similar sensitivities to variations in the the inter-leg angle $\theta$ and stance inclination rate $\dot{\phi}$ (see Table I for the mean values of this error along each of the dimensions).

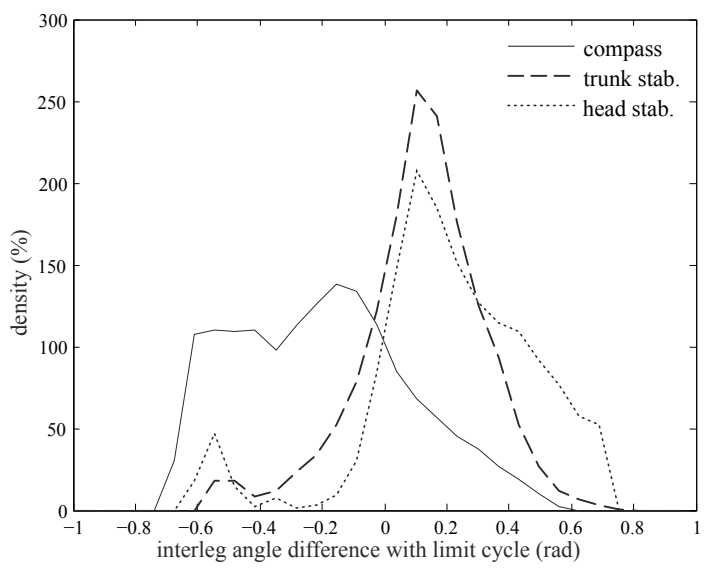

Figure 4. The marginal density, associated to the inter-leg angle $\theta$, of the error between the limit cycle state and the viability kernel states, for the three models, on $4^{\circ}$ slope.

On the contrary, the Fig. 5, related to the inter-leg angle rate $\dot{\theta}$, shows an important difference between upper-body equipped walkers which are centered on the limit cycle and the simple compass which is much more asymmetric around zero, which is confirmed by the Table I. That means that the compass is more sensitive to perturbations in $\dot{\theta}$. In fact, if we reduce the state space to $15 \mathrm{rad} / \mathrm{s}$ around the limit cycle, the number of viable states drops to 24447 for the compass, 23385 for the trunk stabilization and 21615 for the head stabilization. That makes them finally almost equivalent against perturbations on the limit cycle.

Despite the equivalence in the volumes of the viability kernels, this particular sensitivity to $\dot{\theta}$ can make the limit cycle less regular for two-links compass. In order to study this sensitivity, let's analyze the cyclic walking itself, and specifically the stability of this cycle.

Table I

ERRORS BETWEEN VIABLE STATES AND LIMIT CYCLE (MEAN VALUES)

\begin{tabular}{r|ccc} 
& $\theta$ & $\dot{\theta}$ & $\dot{\phi}$ \\
\hline Compass & -0.1679 & 26.2864 & 1.5577 \\
Trunk stabilization & 0.1891 & 1.6704 & 1.0303 \\
Head stabilization & 0.1487 & 2.5020 & 0.8570
\end{tabular}

Table I. We clearly see here that the mean position of $\dot{\theta}$ in the viability kernel of the compass is much farther from the limit cycle than for other walkers.

\section{B. Stability of the cycle}

In the perspective of studying the stability of the dynamical walking, we have chosen to analyze the trajectory of one of the 


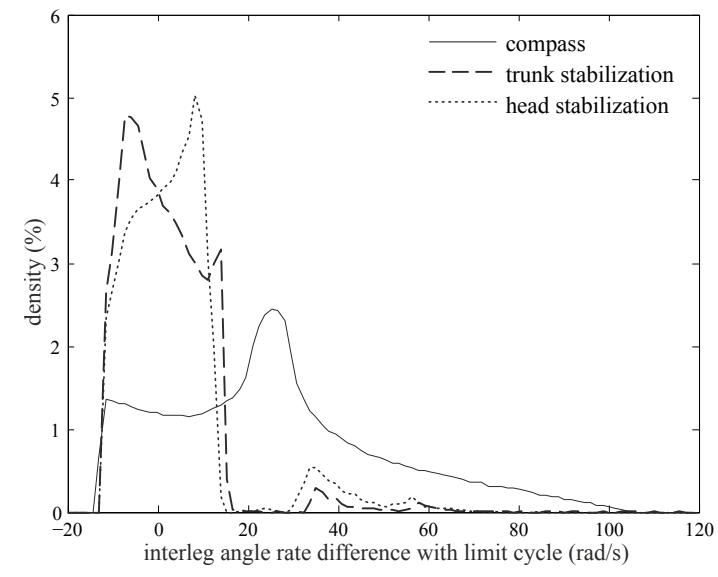

Figure 5. The marginal density, associated to the inter-leg angle variation $\dot{\theta}$, of the error between the limit cycle state and the viability kernel, for the three models, on $4^{\circ}$ slope.

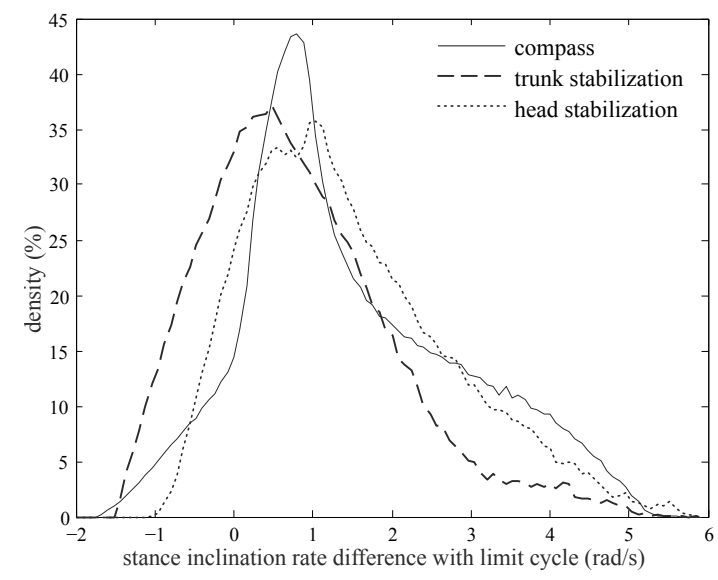

Figure 6. The marginal density, associated to the stance leg inclination rate $\dot{\phi}$, of the error between the limit cycle state and the viability kernel, for the three models, on $4^{\circ}$ slope.

legs of the walkers, let's say it's the left one. We simulate the walking motion, and analyze the trajectory of the inclination $\psi$ of the left leg. In Fig. 7, 8 and 9, the phase plot of limit-cycle left leg inclination is displayed for each of the walker models, when walking during 75 seconds on a $4^{\circ}$ slope (see [13] to help interpreting these plots). These limit-cycles were obtained by simulating a walking motion during 2000 steps before to start the plot. The first observation is that the upper-body walkers have smaller amplitude, in left leg inclinations, than compass one, which means that they make smaller steps. This has already been analyzed as a direct effect of upperbody stabilization [15]. More interestingly, the trajectory for the compass presents a chaotic behavior [13]. This chaos means that the fixed point of the Poincaré map is unstable. We see some trajectories with very small pre-impact swing leg retraction and even impacts that create a discontinuity in the opposite direction to usual ones, which is a sign of an important instability during walking. The only part which is regular is the stance phase, which corresponds to the case where $\psi=\phi$. This is coherent with the previous observation that the compass is less sensitive to the variations of $\phi$ and $\dot{\phi}$ than to variations of $\dot{\theta}$. To analyze this sensitivity, there are dedicated methods such as the Floquet multipliers [16], but they are not adapted to our case because they are not well defined in the case of chaotic behavior.

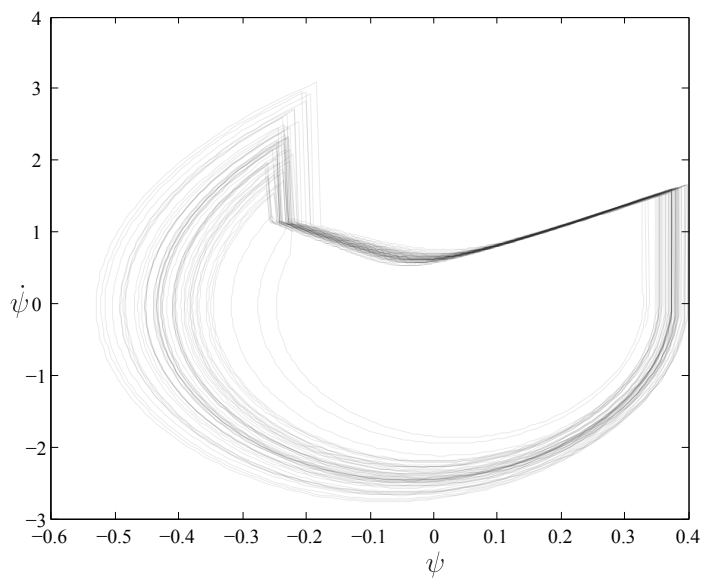

Figure 7. A phase plot of the evolution of left leg inclination of a two-links compass for 75 seconds on a $4^{\circ}$ slope. We see that the limit oscillation is chaotic.

For other walkers, we can see also a chaotic motion, but the amplitudes of the state values is noticeably smaller. we see regular-limit cycles for the left leg inclination. The plots are quite similar, but noticeable differences are visible in post impact trajectories, this shows that trunk stabilization and head stabilization don't deal with impacts in the same way. This the topic of the next and last analysis of this paper.

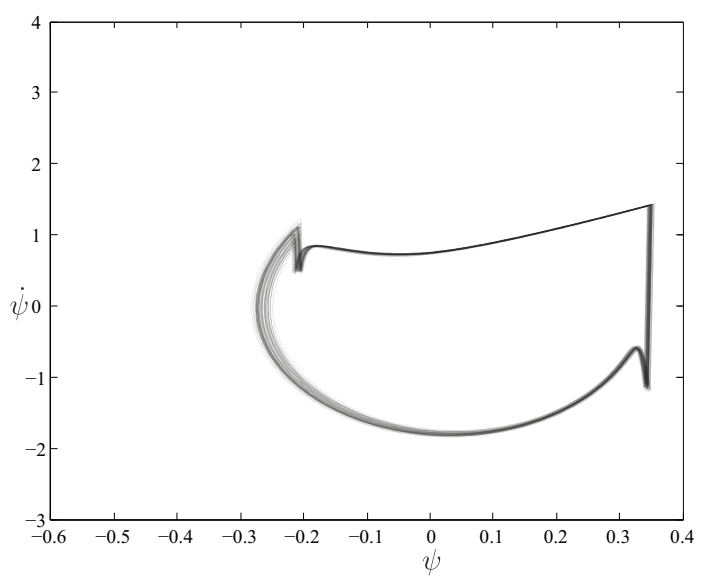

Figure 8. A phase plot of the evolution of left leg inclination of the stabilized trunk walker for 75 seconds on a $4^{\circ}$ slope. The cycle is much more stable. We can see also that the walker maker smaller steps than the compass.

\section{Energy and impacts}

The energy loss in collision is an indicator of the severity of the impacts. The smoothest the impact is, the more efficient is the walking motion, in terms of dissipation of energy. For 


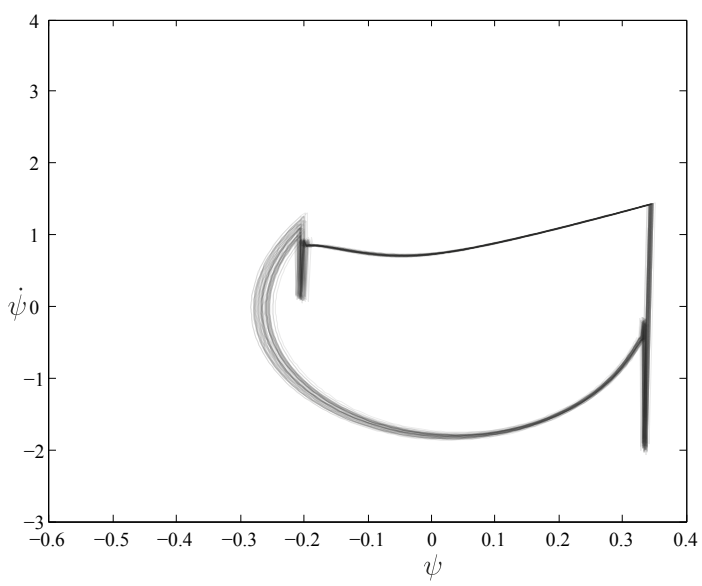

Figure 9. A phase plot of the evolution of one leg inclination of the stabilized head walker for 75 seconds on a $4^{\circ}$ slope. The cycle is similar to the stabilized trunk one, but we notice higher dynamics phases after the impacts.

the same period of 1000 steps, the compass had a mean energy loss of $3.20 \mathrm{~J}$ by step, the trunk stabilization: $1.76 \mathrm{~J}$ and the head stabilization: 1.49J. This is due to the fact that the compass has a CoM up to $26 \mathrm{~cm}$ lower and bigger steps. For illustration, in 1000 steps, the compass traveled for 593 meters, the trunk stabilization walker 536 meters and the head stabilization one made 533 meters. Moreover, there is a part which is absorbed by the actuator itself, the mean power was of $-1.97 \mathrm{~W}$ for the trunk stabilization, and $-3.16 \mathrm{~W}$ for the head stabilization. That means more kinematic energy is absorbed in head stabilization, which explains its $2^{\circ}$ slope disappointing performance in Fig. 3. Nevertheless, the advantage of this property is that impacts are much lighter, which permits better absorption of perturbations during highly dynamical instability, that explains the better performances for steep slopes in Fig. 3.

\section{Discussion AND CONCLUSION}

We have seen throughout this study the effects, in mechanical ways, of the addition of an upper-body and a neck joint to a passive walking system. The upper-body stabilization regulates naturally the limit cycle of these walkers on shallow slopes and improves the balance and perturbation recovery for steeper slopes. However, these features consume the kinematic energy of the walker and require a minimal source of energy, this explains the bad performances of this model in nearly flat grounds.

Our results lead us to believe that adding actuation to the legs is required to overcome directly the energy supply issue. Doing so, upper-body stabilization is expected to contribute to generate stable motions in shallow slopes, and even on rough flat terrain. Furthermore, adding actuation to a passive walker can improve the stability of the walking motion. However, the control of passivity-based walkers is an active research topic that requires a subtle choice of the control strategy to use. This choice is usually a difficult compromise between power efficiency and balance enhancement. This will be the topic of our next study.

This work opens the way to other extensions, for example in terms of mechanical model. We can divide the upper-body into more segments to constitute a vertebral column. Our results suggest that a more complex upper-body can improve again the stability of walking motions.

\section{Media ATtACHMENT}

The attached video shows (A) a simulation of the limitcycles for the three walkers on $4^{\circ}$ slope, with a speed-up to better notice irregularities, particularly in the two-links compass case. And (B) examples of slope inclination changes that the upper-body walkers overcome and not the compass.

\section{ACKNOWLEDGMENT}

This work is partially supported by grants from the EU Project CLONS (Closed-Loop Neural Prosthesis for Vestibular Disorders, Grant Agreement 225929).

\section{REFERENCES}

[1] P.-B. Wieber, "Viability and Predictive Control for Safe Locomotion," in IEEE-RSJ IROS, Nice, France, 2008.

[2] S. Collins, A. Ruina, R. Tedrake, and M. Wisse, "Efficient bipedal robots based on passive-dynamic walkers," Science, vol. 307, no. 5712, pp. 1082-1085, February 2005

[3] S. H. Collins, M. Wisse, and A. Ruina, "A three-dimensional passivedynamic walking robot with two legs and knees," International Journal of Robotic Research, vol. 20, pp. 607-615, 2001.

[4] A. Goswami, B. Espiau, and A. Keramane, "Limit Cycles in a Passive Compass Gait Biped and Passivity-Mimicking Control Laws," Autonomous Robots, vol. 4, pp. 273-286, 1997.

[5] H. Hicheur, S. Vieilledent, and A. Berthoz, "Head motion in humans alternating between straight and curved walking path: Combination of stabilizing and anticipatory orienting mechanisms," Neuroscience Letters, vol. 383, pp. 87-92, 2005.

[6] I. Farkhatdinov, H. Michalska, A. Berthoz, and V. Hayward, "Modeling verticality estimation during locomotion," in Proc. of the CISM-IFToMM Symposium on Robot Design, Dynamics, and Control., 2012.

[7] B. Kaddar, Y. Aoustin, and C. Chevallereau, "Arms swing effects on a walking planar biped," Institut de Recherche en Communications et en Cybernetique de Nantes (IRCCyN), Tech. Rep., 2012.

[8] M. Wisse, D. Hobbelen, R. Rotteveel, S. Anderson, and G. Zeglin, "Ankle springs instead of arc-shaped feet for passive dynamic walkers," IEEE-RAS Humanoids, pp. 110-116, Dec 2006.

[9] C. Chevallereau, J. Grizzle, and C.-L. Shih, "Asymptotically stable walking of a five-link underactuated 3-d bipedal robot," IEEE-TRo, vol. 1 , no. 25 , pp. 37-50, January 2009.

[10] M. Wisse, D. G. E. Hobbelen, and A. L. Schwab, "Adding an upper body to passive dynamic walking robots by means of a bisecting hip mechanism," IEEE-TRo, vol. 23, no. 1, pp. 112 -123, feb. 2007.

[11] F. Asano and Z.-W. Luo, "Pseudo virtual passive dynamic walking and effect of upper body as counterweight," in IEEE/RSJ IROS, sept. 2008, pp. $2934-2939$

[12] T. McGeer, "Stability and control of two-dimensional biped walking," Simon Fraser University, Centre for Systems Science, Burnaby, B.C., Canada, Tech. Rep., 1988.

[13] A. Goswami, B. Thuilot, and B. Espiau, "Compass-Like Biped Robot Part I : Stability and Bifurcation of Passive Gaits," INRIA, Research Report RR-2996, 1996.

[14] H. G. Armstrong, "Anthropometry and mass distribution for human analogues. volume 1. military male aviators," Aerospace Medical Research Lab Wright-Patterson AFB Ohio USA, Tech. Rep., March 1988.

[15] Y. Hoshino, C. Fu, and K. Chen, "A passive walking strategy for a biped robot with a large mass torso by a spring and a damper," in ICMA, aug. 2011, pp. $1269-1274$.

[16] D. G. E. Hobbelen and M. Wisse, "Limit cycle walking," in Humanoid Robots: Human-like Machines, 2007, pp. 277-294. 